\title{
CLASSIFICATION OF BRAIN TUMOR USING BEES SWARM OPTIMISATION
}

\author{
M. Ramkumar' ${ }^{1}$ M. Babu' ${ }^{2}$ and R. Lakshminarayanan ${ }^{3}$ \\ ${ }^{1,3}$ Department of Computer Science and Engineering, Gnanamani College of Technology, India \\ ${ }^{2}$ Department of Computer Science and Engineering, Sri Satya Sai University of Technology and Medical Sciences, India
}

\begin{abstract}
Nowadays, processing the medical image is a most significant diagnostic process. Usually RMI is used to detect the presence of and type of tumor. The following process is very complicated in the brain tumor classification. The treatment of medical images, such as image segmentation, image extraction, and image classification, takes various steps. Various types of properties such as intensity, forms and texturebased features are extracted from a segmented MRI image. The feature selection approach is employed to select a small subset of MRI image features that minimize redundancy and maximize target-related pertinence. This article uses the Bees Swarm Optimization (BSO) for the selection and the Neural Network Classifier to classify the type of tumor in present brain MRI images, and then takes online MRI images which contain brain tumor, then a machine-learning model.
\end{abstract}

Keywords:

Neural Network, ACO, Feature Extraction, Classification, MRI

\section{INTRODUCTION}

Brain tumor is an abnormal and undesirable growth of tissue cells in brain which leads to certain neurological disorder for human. Now a days due to environmental and human life style these tumor cases are rapidly increasing [1]. To cope with this situation, we require combination of a computer aided diagnosis (CAD) system and a medical imaging technique which generate very high quality images of the infected body part mainly soft tissues of human beings. Magnetic resonance imaging (MRI) is a imaging technique generally used for brain to provide relevant information, based on which physician or CAD can diagnose whether patient have any tumor or not, if there is tumor detected then they can further differentiate between its types so that proper treatment can be given to that patient. MRI depict every required details without radiation unlike X-ray imaging [2]. It is flexible method since a contrast between one tissue and other, can be changed by altering the way of imaging. For instance, by changing the radio frequency and gradient pulses it is possible to produce images with high contrast. Brain tumor mainly in two categories benign and malignant [3]. Benign tumor is noncancerous while malignant tumor is more prone to be cancerous which may be developed because of cancer at any part of the body not just only brain. The Fig. 1 shows all the cases.

Medical image processing has provided lots of techniques which deals to automate this task in less time and with more accuracy. Feature extraction and selection, Image segmentation and Image classifications are the very important steps in medical image processing. Feature selection is even more important than feature extraction because optimal feature subset is deemed necessary to improve the image classifier performance and decrease the computation time. In traditional approaches we have Principal Component Analysis (PCA) and Linear Discriminant Analysis (LDA) for linear, and KernalPCA has been used for nonlinear features [3] [4]. In compare to these type of method Evolutionary computation (EC) techniques gives better results [5].

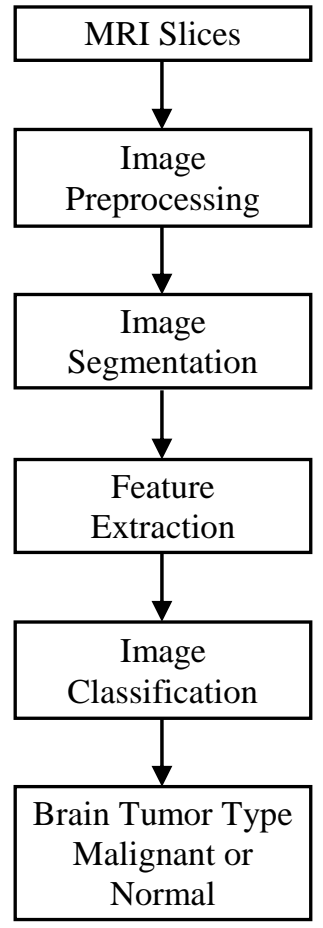

Fig.1. Medical Image Processing Steps

\section{RELATED WORKS}

Various techniques have in the past been used to predict and classify the tumor from MRI brain images. As discussed in section 1, PCA is mainly intended for the reduction and selection of features due to its simplicity, but can only be used for linear features over the features selected on part of the top main components. During this process, several important features are therefore ignored and numerous features are included in the feature subset [8]. For tumor reduction [9], Gladis et al. [11] used the combination PCA and LDA [11].

EC algorithms have been used recently mainly for the selection of functionalities because limitations found in traditional techniques are overcome by them. For the selection of the main space for the sub-set function, genetic Algorithm (GA) can be applied. With the minor flaw of inability to identify the world's optimum and unable to solve variant problems, the GA produces much greater results than the PCA [10] [11]. BSO is proposed and implemented to select optimal features in order to solve this problem. The search method for BSO is metaheuristic and bio-inspired [12] [13]. 


\section{PROPOSED METHOD}

Medical image processing with a CAD system is used to diagnose brain tumor. There are several steps used in paper before the brain tumor is classified when MRI is given. These steps are shown in the Fig.1.

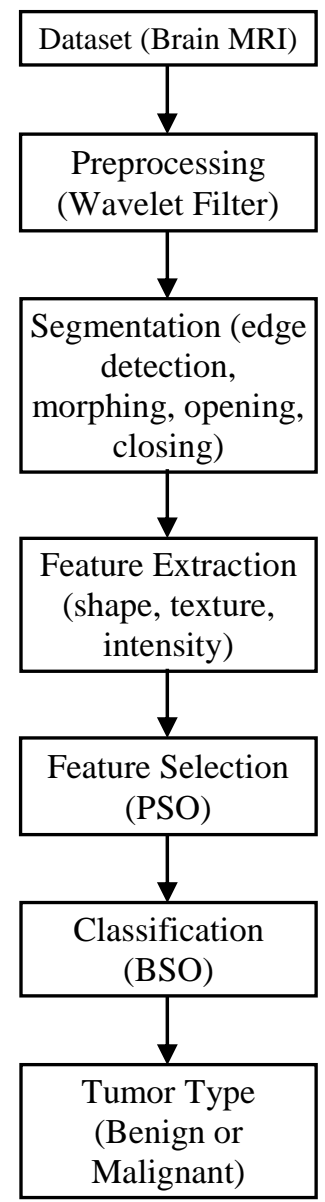

Fig.2. Proposed Research Methodology

\subsection{IMAGE PREPROCESSING}

After an MRI image is taken as an input image it takes several steps before the image segmentation process takes place. We must first convert this picture into a gray scale picture and then we remove noise or inhomogeneity from it.

\subsection{IMAGE SEGMENTATION}

The process of dividing the image into a different homogeneous part is image segmentation. In this case, the part of the brain tumor must be segmented from the entire brain MRI that is our region of interest.

\subsection{FEATURE EXTRACTION AND SELECTION}

Feature Extraction is the procedure for collecting initial data and then collecting relevant information from so-called feature vectors as to allow these features to be used as a vector for further processing instead of entire data. Feature selection or reduction is a technique in which we select certain features from a number of features that were extracted because this feature alone affects the classification process.

The PSO is a random search process based on the population. We assume that $N$ particles appear randomly in a solution space. Note that we solve the optimization problem and for the clustering of data, for each particle at its position in the solution space, there are always criteria (e.g. squared error function). The $N$ particles will continue to move and calculate the criteria at each stay position (we call fitness in PSO).

In the solution space that is associated with the most appropriate solution (fitness) achieved by the solution particle, each particle keeps track of its co-ordinates. This is the best personal value, pbest.

The PSO other best value is the best value any particle in the neighborhood has ever achieved. This is known as the best global value.

We introduce the exact statement in mathematics below:

$$
\begin{aligned}
& v_{i, j}(t)=w \cdot v_{i, j}(t-1)+c_{1} \cdot r_{1}\left(p_{i, j}(t-1)\right. \\
&\left.-x_{i, j}(t-1)\right)+c_{2} \cdot r_{2}\left(p_{g, j}(t-1)\right. \\
&\left.-x_{i, j}(t-1)\right) \\
& x_{i, j}(t)=x_{i, j}(t-1)+v_{i, j}(t)
\end{aligned}
$$

where $x_{i}$ is the current position of the particle, $v_{i}$ is the current velocity of the particle, $p_{i}$ is the personal best position of the particle, $w, c$, are all constant factors, and $r$ are the random numbers uniform distributed within the interval $[0,1]$.

We now use last speed and the last personal and worldwide position to predict the speed. The position we remain is now determined by the final position plus the speed.

By using PSO the initial "K meaning" problem can be solved and the whole partial clustering system still maintained. The most important thing is to consider it as a problem of optimization.

\subsection{IMAGE CLASSIFICATION}

Image classification means that we categorize any pixel of an image into a class e.g. any remote satellite image in the earth, and we can classify any pixel according to its characteristics, regardless of whether it belongs to the water body area or the land area. Computers can likewise classify themselves if they are trained in different class features [17]. This training can be monitored or unattended. If the computer predicts the tumor, it also divides the tumor into benign and malignant.

\subsubsection{Bees Swarm Optimisation:}

Swarm comportament (fish schools, birds ' flocks, land herds, communities of insects, etc.) is based on people's biological needs to stay together. In this way, the chances of people remaining alive are increased because predators usually only attack isolated people. Collective movement is characteristic of flocks of birds, herds of animals and fish schools. Swarm behavior is also characterized by colonies of diverse social insects (bees, wives, ants, termites). The swarm behavior is characterized primarily by independence, distribution and self-organization. The systems of communication between insects contribute to the "swarm intelligence" pattern of collective intelligence. 
Swarm Intelligence is the Artificial Intelligence branch that investigates actions of individuals in various decentralized systems. The Multi-agent Systems are made up by individuals who communicate, cooperate, share information and knowledge and perform certain tasks in their environment (e.g. robots) or by "virtual" (artificial). Researchers use certain principles of natural swarm intelligence to develop Swarm Intelligence models. The development of artificial systems usually does not involve the whole picture of natural systems, but explores and adapts them in the quest for ideas and models.

Bees seek food in nature by exploring the fields in the hive district. The food is collected and collected by other bees for later use. Usually some scouts look for the region in the initial step. At the end of the search, the scout bees return to the hive and inform the scout bees on their food sources in the areas examined about their places, quantity and quality. If they have discovered nectar in previously examined places, scout bees are dancing in the socalled "dance floor" area of the hive to "advertise" food places and encourage the remainder to follow. The information on the amount of food is provided by a ritual called a « dance of the wagon ». If a bee decides to leave the hive to gather nectar, then one of the scout bees follows a patch of flowers that was previously found. When they arrive, the fodder bee takes nectar and comes back to the hive to abandon the nectar to a food shop. A foraging bee can be subjected to several scenarios:

- It can abandon the food location and return to its role of an uncommitted follower;

- It can continue with the foraging behavior at the discovered nectar source without recruiting the rest of the colony;

- It can try to recruit its hive-mates with the dance ritual before returning to the food location.

The bee chooses one of the options above. Since several bees are trying to recruit their hive-mates simultaneously in a dancing floor area, it is not clear how a non-binding bee decides to follow which one. The only obvious fact is that "the loyalty of bees always depends on the quantity and quality of the source of food." The process described continues on and off with the bees accumulating nectar and exploring new areas with a potential source of food.

The main steps of any honeybees ' algorithm are: forage and waggle dance. The role of the waggle dance (the informationexchange) is to examine the quality of existing solutions and direct them towards the new ones. Foraging is the solution creation stage. The idea of developing these algorithms was based on simple rules for modeling the nectar collection organized.

The following algorithms are similar between the way bees look for food in nature and the way optimization algorithms are searching for the best possible combination of optimization problems. The main idea was to create a multi-agent system to solve difficult combinational optimization problems efficiently. Through the search room the artificial bees explore possible solutions. Artificial bees cooperate and exchange information to enhance the quality of the solutions found. The artificial bees focus on more promising fields by collective knowledge and information exchange and gradually discard less promising solutions.

BSO's fundamental idea is to build a multi agent system (colony of artificial bees), that will find good solutions to various problems in combinatorial optimisation and explore the principles used in the nectar collection process by honey beasts. A small number of individuals usually consist of artificial bee colony but the BSO principles are nevertheless collected from natural systems. The search room for feasible solutions is used by artificial bees. Autonomous artificial bees work together and exchange information to find the best solutions possible. Artificial bees are concentrating on the more promising areas and slowly leaving the less promising areas with their collective knowledge and information sharpening. Gradually, artificial bees generate and/or improve solutions collectively. The BSO search is carried out in iteration until certain predefined stop criteria are fulfilled.

The authors developed two different approaches during the evolution of the BSO algorithm. In the first approach, bees build solutions part by part, based on constructive steps. The second approach, a very real one in the BSO algorithm, is to improve complete solutions in order to achieve the best final solution. It's called a BSO approach. In the next text, the general description of the algorithm explains both concepts.

The population of individual agents (artificial bees) is BSO. The problem is resolved by each artificial bee. The BSO algorithm consists of two alternating phases (Forward Pass and Reverse Pass). All artificial bees explore the search area on each forward pass. They use a predefined number of steps that build/improve partial or full solutions and produce the new partial/full solutions.

Algorithm for classification

Step 1: Initialise:

Step 2: Find number of bees, percentage of experienced foragers, scouts, onlookers, dimension, radius and end condition

Step 3: For all the bees

a. Initialise the bees randomly inside the search space

b. Do

c. Compute fitness of bees

d. Sort bees using its fitness value

e. Partition the swarm into the experienced forager, onlooker and scout

Step 4: end

Step 5: For all the experienced forager bees

a. For D-dimensional search space

b. update the previous best position

c. select elite bee for all the experienced forager bees

i. For update the position of an experienced forager bee

ii. end

d. end

Step 6: end

Step 7: For each onlooker bee

a. select an elite bee from experienced forager bee for onlooker

b. For D-dimensional search space

i. update the position of an onlooker bee

c. end

Step 8: end

Step 9: For each scout bee 
a. For D-dimensional search space

i. walk randomly around the search space

b. end

Step 10: end

Step 11:adjust the radius and step size of the search space for scout bees

Step 12: end until the termination criterion is met

\section{RESULTS AND DISCUSSIONS}

No tumor detection is performed by medical image processing steps in the brain MRI image. In total, 354 brain MRI pictures were taken from the online BRATS-2015 database by several patients. Of the 78 theses, the picture has no malignant or benign tumor and the rest has tumor. For entire experiments, Matlab 2016a was used. Wavelet filter was used to remove noise in MRI images of any kind [21]. The tumor region is subsequently segmented from MRI by using soft edge detection techniques and by applying morphological operations. Then a total of 14 features from the shape, texture and intensity class are extracted. Then these 14 features are only selected by using the BSO algorithm to help with classification. These functions are finally used to train the neural network classifier in order to detect the type of tumor, which is less than the total functionality.

In the given dataset, we have two different kinds of tumors benign and malignant. We compare the accuracy of the classification system with the case where each feature has been used after training neural network classification with the feature selection performed by BSO. The Fig. 3 shows the specification and specification. In Fig.4 and Fig.5, a total of 6 out of 14 selected BSO features appear in Table.1. The selected features are represented by 1 and the rejected features by 0 . The Fig. 6 and Fig.7 shows benign and malignant tumors of both classified tumors. The result shows that $95.23 \%$ of classification accuracy with hybrid BSO-neural network model is better than the simple $86.82 \%$ neural network classifier.

Table.1. Total Features

\begin{tabular}{|l|c|c|}
\hline Name of feature & All feature & BSO selected feature \\
\hline Area & 1 & 1 \\
\hline Perimeter & 1 & 0 \\
\hline Circularity & 1 & 1 \\
\hline Mean & 1 & 0 \\
\hline Standard Deviation & 1 & 0 \\
\hline Variance & 1 & 1 \\
\hline Skewness & 1 & 0 \\
\hline Kurtosis & 1 & 1 \\
\hline Entropy & 1 & 1 \\
\hline Contrast & 1 & 0 \\
\hline Correlation & 1 & 0 \\
\hline Energy & 1 & 1 \\
\hline Homogeneity & 1 & 0 \\
\hline IDM & 1 & 0 \\
\hline
\end{tabular}

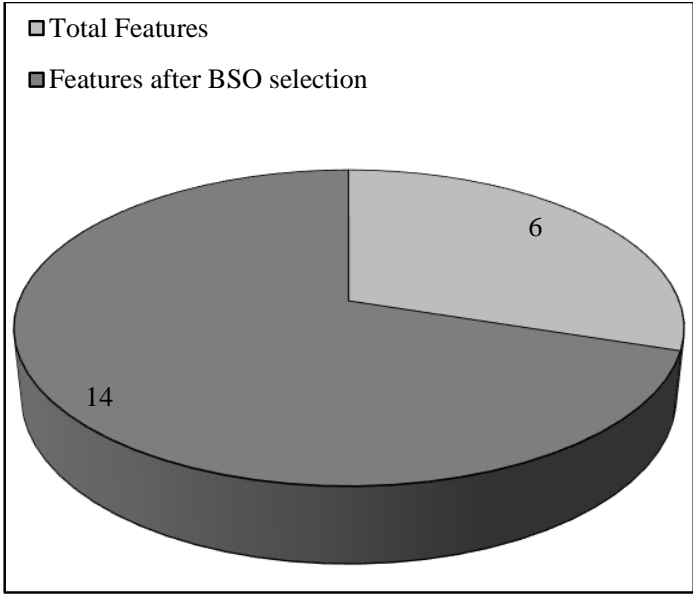

Fig.3(a). Number of features selected

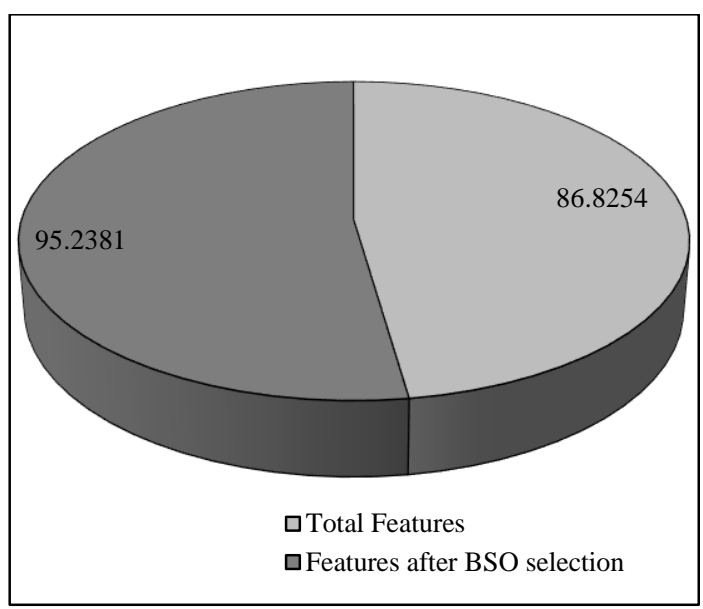

Fig.3(b). Accuracy of features selected

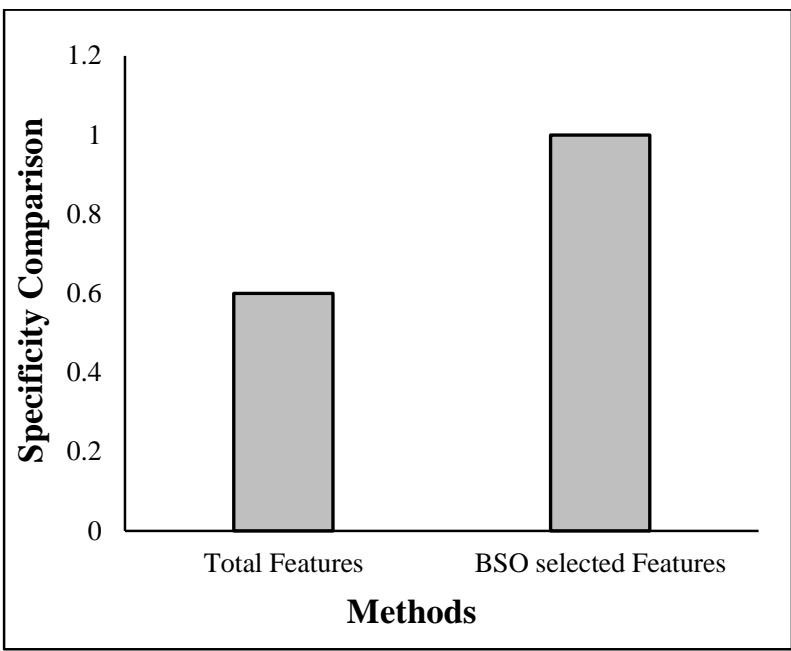

Fig.4. Specificity 


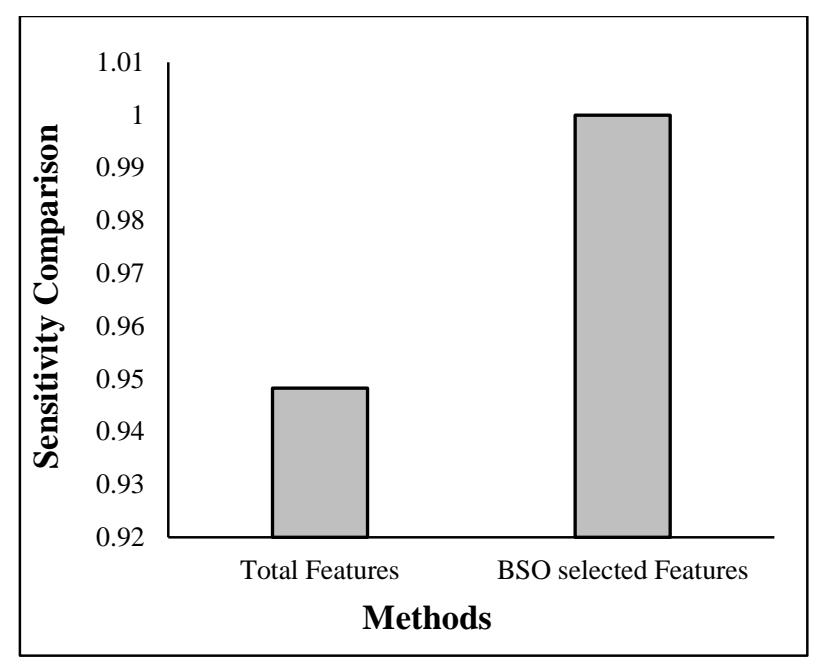

Fig.5. Sensitivity

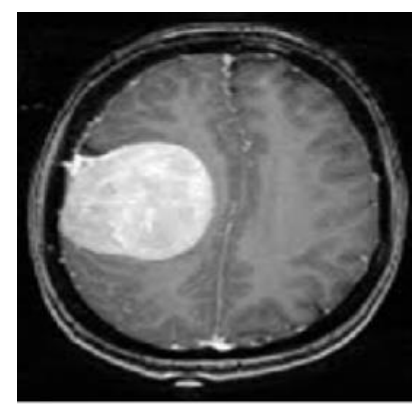

Brain MRI Image

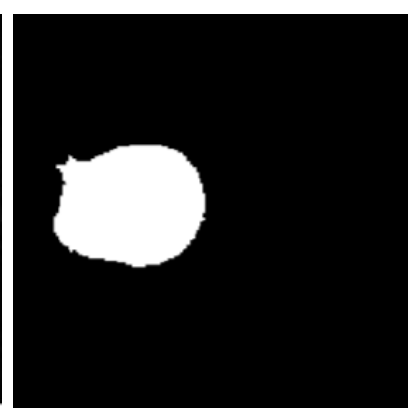

Segmented image
Fig.6. Benign Tumor Classification

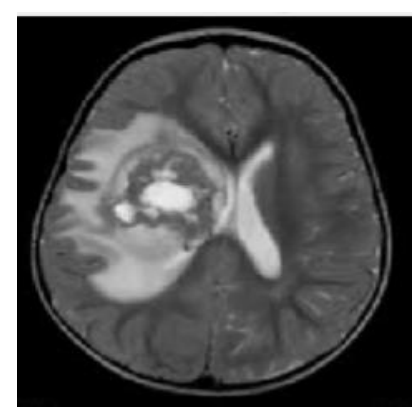

Brain MRI Image

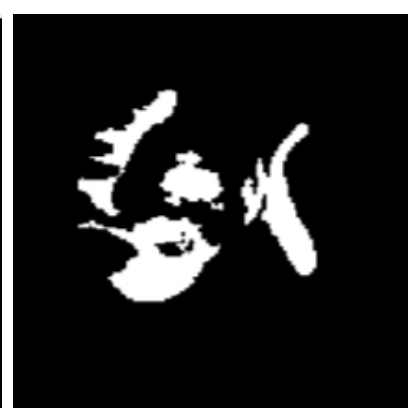

Segmented image
Fig.7. Malignant Tumor Classification

\section{CONCLUSIONS}

We have used in this paper the hybrid BSO-NN model for the classification of brain tumors. We choose minimum required features using bio-inspired algorithms called BSO instead of taking all features. Fewer features, but all that are important, lead to the accuracy of BSO's classification. The use of key features only increases efficiency and reduces computing time. By using the BSO-NN classification model the accuracy of simple NN classification is $95.23 \%$ compared to $86.82 \%$ if all 14 functions are included. Similarly, $94.8 \%$ of specificity and $100 \%$ of sensitivity. In the future we want to apply concept and compare all of these with different types of medical images with other organically inspired algorithms.

\section{REFERENCES}

[1] A. Devi, A. Babu, A.K. Menon and R.R. Chaandran, "Brain Tumor Detection", International Journal of Innovative Technology and Research, Vol. 3, No. 2, pp. 1950-1952, 2015.

[2] Debnath Bhattacharyya and Tai-Hoon Kim, "Brain Tumor Detection using MRI Image Analysis", Proceedings of International Conference on Ubiquitous Computing and Multimedia Applications, pp. 1-6, 2011.

[3] Y. Zhang and L. Wu, "An MR Brain Images Classifier Via Principal Component Analysis and Kernel Support Vector Machine", Progress in Electromagnetics Research, Vol. 130, pp. 369-388, 2012.

[4] A.K. Jain, R.P.W. Duin and J. Mao, "Statistical Pattern Recognition: A Review", IEEE Transactions on Pattern Analysis and Machine Intelligence, Vol. 22, No. 1, pp. 3137, 2000.

[5] K. Deb and A.R. Reddy, "Reliable Classification of Two Class Cancer Data using Evolutionary Algorithms", BioSystems, Vol. 72, No. 1-2, pp. 111-129, 2003.

[6] J. Kennedy and R. Eberhart, "Particle Swarm Optimization", Proceedings of International Conference on Neural Networks, pp. 1942-1948, 1995.

[7] S.A. Taie and W. Ghonaim, "CSO-based Algorithm with Support Vector Machine for Brain Tumor Disease Diagnosis", Proceedings of International Workshops on Pervasive Computing and Communications, pp. 332-343, 2017.

[8] M.A. Majid, A.F.Z. Abidin, N.D.K. Anuar, K.A. Kadiran, M.S. Karis, Z.M. Yusoff, N.H.K. Anuar and Z.I. Rizman, "A Comparative Study on the Application of Binary Particle Swarm Optimization and Binary Gravitational Search Algorithm in Feature Selection for Automatic Classification of Brain Tumor MRI", Journal of Fundamental and Applied Sciences, Vol. 10, No. 2, pp. 486-498, 2018.

[9] V.P. Gladis, Pushpa Rathi and S. Palan, "Brain Tumor MRI Image Classification with Feature Selection and Extraction using Linear Discriminant Analysis", Proceedings of International Conference on Computer Vision and Pattern Recognition, pp. 233-238, 2012.

[10] B. Xue, M. Zhang and W.N. Browne, "Particle Swarm Optimization for Feature Selection in Classification: A Multi-Objective Approach", IEEE Transactions on Cybernetics, Vol. 43, No. 6, pp. 68-73, 2013.

[11] Balasubramanian Vijaya Lakshmi and Vasudev Mohan, "Kernel-based PSO and FRVM: An automatic plant leaf type detection using texture, shape, and color features", Computers and Electronics in Agriculture, Vol. 125, pp. 99112, 2016.

[12] N. Zulpe and V. Pawar, "GLCM Textural Features for Brain Tumor Classification”, International Journal of Computer Science, Vol. 9, No. 3, pp. 354-367, 2012.

[13] Atiq Ur Rehman, Aasia Khanum and Arslan Shaukat, "Hybrid Feature Selection and Tumor Identification in Brain MRI Using Swarm Intelligence", Proceedings of IEEE 
International Conference on Frontiers of Information Technology, pp. 441-448, 2013.

[14] Rajaguru Harikumar and Sunil Kumar Prabhakar, "Oral Cancer Classification from Hybrid ABC-PSO and Bayesian LDA", Proceedings of IEEE International Conference on Communication and Electronics Systems, pp. 1-7, 2017.

[15] N.N. Gopal and M. Karnan, "Diagnose Brain Tumor through MRI using Image Processing Clustering Algorithms such as Fuzzy C Means along with Intelligent Optimization Techniques", Proceedings of IEEE International
Conference on Computational Intelligence and Computing Research, pp. 26-34, 2010.

[16] P. Vivekanandan, "An Efficient SVM based Tumor Classification with Symmetry Non-Negative Matrix Factorization using Gene Expression Data", Proceedings of IEEE International Conference on Information Communication and Embedded Systems, pp. 761-768, 2013.

[17] G. Jothi and H. Inbarani, "Hybrid Tolerance Rough SetFirefly based Supervised Feature Selection for MRI Brain Tumor Image Classification", Applied Soft Computing, Vol. 46, pp. 639-651, 2016. 